\title{
Genetic mapping and QTL analysis of Botrytis resistance in Gerbera hybrida
}

\author{
Yiqian Fu • Alex van Silfhout • Arwa Shahin • Ronny Egberts • Martin Beers • \\ Ans van der Velde • Adrie van Houten • Jaap M. van Tuyl • Richard G. F. Visser • \\ Paul Arens $(\mathbb{D}$
}

Received: 16 September 2016/Accepted: 25 December 2016 / Published online: 23 January 2017

(C) The Author(s) 2017. This article is published with open access at Springerlink.com

\begin{abstract}
Gerbera hybrida is an economically important cut flower. In the production and transportation of gerbera with unavoidable periods of high relative humidity, grey mould occurs and results in losses in quality and quantity of flowers. Considering the limitations of chemical use in greenhouses and the impossibility to use these chemicals in auction or after sale, breeding for resistant gerbera cultivars is considered as the best practical approach. In this study, we developed two segregating F1 populations (called S and F). Four parental linkage maps were constructed using common and parental specific SNP markers developed from expressed sequence tag sequencing. Parental genetic maps, containing 30, 29, 27 and 28 linkage groups and a consensus map covering 24 of the 25 expected chromosomes, could be constructed. After evaluation of Botrytis disease severity using three different tests, whole
\end{abstract}

Electronic supplementary material The online version of this article (doi:10.1007/s11032-016-0617-1) contains supplementary material, which is available to authorized users.

Y. Fu • A. van Silfhout - A. Shahin · J. M. van Tuyl •

R. G. F. Visser • P. Arens $(\triangle)$

Plant Breeding, Wageningen University \& Research, PO Box 386, 6700AJ Wageningen, The Netherlands

e-mail: paul.arens@wur.nl

R. Egberts · A. van Houten

Schreurs Holland B.V., Hoofdweg 81, 1424PD De Kwakel,

The Netherlands

M. Beers - A. van der Velde

Florist Holland B.V., Dwarsweg 15, 1424PL De Kwakel,

The Netherlands inflorescence, bottom (of disc florets) and ray floret, quantitative trait locus (QTL) mapping was performed using the four individual parental maps. A total of 20 QTLs (including one identical QTL for whole inflorescence and bottom tests) were identified in the parental maps of the two populations. The number of QTLs found and the explained variance of most QTLs detected reflect the complex mechanism of Botrytis disease response.

Keywords Gerbera grey mould · SNP. Linkage group · QTL mapping

\section{Introduction}

Gerbera hybrida belongs to the Compositae family and is known for its abundant flower colours, capitula sizes and shapes. As one of the most economical important ornamental plants, gerbera is mainly used as cut flower and ranked fifth in cut flower sales on Dutch flower auctions in 2014 (https://www.floraholland. com/media/3949227/Kengetallen-2014-Engels.pdf). The cultivated commercial gerberas are highly heterozygous, almost completely obligatory outcrossing diploid $(2 n=50)$ plants and probably originated from crossings between two wild species, Gerbera jamesonii and Gerbera viridifolia, from Africa (Hansen 1999).

Gerbera as a cut flower is mainly grown in greenhouses for year-round production. However, during gerbera cultivation especially in winter and during the process of post-harvest transportation, the high relative 
humidity is ideal for grey mould infestation. Gerbera grey mould is caused by the necrotrophic fungus Botrytis cinerea, a notorious fungal pathogen with a wide range of plant host species (Elad et al. 2016). B. cinerea infection leads to direct damage on gerbera. Necrotic lesions (spotting) in early infection occur on flower buds and ray florets and these symptoms are strengthened when flowers are packed in boxes, in which a high relative humidity develops during cold storage and transport (Bastiaan-Net et al. 2007; Kerssies 1993a; Kerssies 1993b; Salinas and Verhoeff 1995). Control of gerbera grey mould in greenhouses frequently relies on spraying chemicals (Prins et al. 2000), but using chemicals may cause environmental issues and increase resistance to fungicides (Leroux 2007) whereas the use of some compounds has been restricted and banned in a number of countries. Moreover, quality loss due to gerbera grey mould occurring in post-harvest (in auction or after sale) transport is hard to avoid by chemical treatments, affecting both the buyer (reduction in profits) and the seller (a breeder's reputation) (Bastiaan-Net et al. 2007). Thus, breeding for Botrytis resistant varieties is needed to reduce current and future problems due to this devastating pathogen in gerbera.

A number of studies on Arabidopsis indicated that the positive responses regulated by JA/ET (jasmonic acid/ethylene) signalling (Glazebrook 2005; Thomma et al. 1998; Thomma et al. 1999) and production of camalexin (Kliebenstein et al. 2005; van Baarlen et al. 2007; Williamson et al. 2007) enhance a plant's resistance to B. cinerea. Similarly, in Brassica rapa, glucosinolate defensive metabolite accumulation coincided with $B$. cinerea quantitative trait loci (QTLs) (Zhang et al. 2016). Catechol from onion scales inhibited $B$. cinerea growth in vitro (Clark and Lorbeer 1975). However, none of these metabolites in different species can confer full resistance. Plant resistance to Botrytis is considered conditioned by multiple genes with partial effects and likely requires the contribution of multiple loci to reduce disease severity (Mengiste et al. 2003) and to obtain acceptable levels of resistance under standard conditions. This kind of complex resistance is polygenic and can be referred to as quantitative disease resistance (St. Clair 2010). DNA markers tightly linked to quantitative resistance loci can be used for marker-assisted selection (MAS) and desirable QTLs can be then subsequently introgressed into commercial cultivars.

Up to now, QTL analysis for Botrytis resistance has been primarily assayed in Arabidopsis and tomato.
Denby et al. (2004) identified 12 small- to mediumeffect QTL governing Botrytis susceptibility as to lesion size in Arabidopsis using 104 individuals from a Ler $\times$ Col-0 recombinant inbred population and several interesting candidate genes were found co-located in the QTL regions of the genome. Rowe and Kliebenstein (2008) found that several separate QTLs influenced lesion size and camalexin accumulation on Arabidopsis leaves using a larger RILs population with 411 individuals. They suggested that the plant defence against $B$. cinerea is mainly quantitative and genetically complex. Finkers et al. (2007b) calculated disease incidence and lesion growth rate in tomato populations and detected three QTLs that explained 12, 15 and $7 \%$ of the total phenotypic variation. They also analysed two QTLs in $\mathrm{BC} 2 \mathrm{~S} 1$ progeny and found additive effects for progeny with homozygous resistance QTL alleles present.

No gerbera genetic maps are published to date. In this study, we developed two F1 populations segregating for Botrytis resistance in order to obtain the first genetic maps for this highly heterozygous ornamental crop. Through next generation sequencing of the transcriptomes of the parental genotypes ( $\mathrm{Fu}$ et al. 2016), SNP markers have been developed. These SNP markers have been used for linkage map construction and QTL mapping of Botrytis resistance in gerbera.

\section{Materials and methods}

\section{Mapping populations}

Two gerbera segregating F1 populations from heterozygous parents were used in this study. The two mapping populations were derived from four parental genotypes with different resistance levels against $B$. cinerea infection and the selected (unrelated) two populations showed the largest variation among $20 \mathrm{~F} 1$ populations (four half sibs of crosses with a line with known Botrytis infection problems) which were tested for Botrytis susceptibility on 50 individuals. Population Schreurs (hereafter referred to as population S), containing 276 offspring, was obtained from a cross between the gerbera genotypes, SP1 and SP2. Population Florist (hereafter referred to as population F) was produced by a cross between FP1 and FP2. Population F consisted of 270 progeny. All individuals from both populations were used for linkage mapping, disease tests and QTL analysis. 
Phenotypic measurements

The head-like inflorescence of gerbera is composed of different flower types, the marginal ray florets, the central disc florets and the intermediate trans florets. Botrytis-infected lesion symptoms vary in these gerbera florets: spotting on ray florets and rotting on disc florets. To assess Botrytis resistance levels on different gerbera inflorescences of all $\mathrm{F} 1$ progenies and four parents in the two populations, phenotypic data were collected using three tests based on a visual inspection of Botrytis infestation: on whole inflorescence (further referred to as whole inflorescence or WI test), on the bottom of disc florets in the capitulum (further referred to as bottom test) and on ray florets (further referred to as ray floret or $R F$ test), respectively.

B. cinerea (strain B05.10 obtained from Dr. J. van Kan, Laboratory of Phytopathology, Wageningen University) was grown for 1 week on potato dextrose agar (PDA) medium after which conidia were transferred onto fresh PDA medium and grown until sporulation (about 1 week). A spore suspension of $1 \times 10^{7}$ conidial spores per millilitre in sterile distilled water was prepared as stock suspension. For the Botrytis disease test on whole inflorescence and bottom, the spore suspension was diluted to a concentration of $1 \times 10^{5} / \mathrm{ml}$ with water and sprayed on the inflorescence with a fine plant sprayer. After inoculation, inflorescences were incubated for 5 days in a climate cell at $20{ }^{\circ} \mathrm{C}$ and a R.H. of $90 \%$. Because ripe flowers (anthesis of first whorl of disc floret) are not available from single plants in abundance, testing was done over a period of 10 consecutive weeks (8-10 inflorescences tested on average). Inflorescence testing was done simultaneously for whole inflorescence and bottom tests on the same inflorescence. First, whole inflorescences were visually evaluated to score, after which, the bottom of the capitulum was cut (horizontal cross section) to check (score) fungal growth inside the capitulum for the bottom test. The response to Botrytis infection on whole inflorescence and bottom was scored ranging from 0 (no symptom) to 5 (completely rotten).

For the ray floret test, inoculation was performed by pipetting $2 \mu \mathrm{l}$ of spore suspension that was diluted to a concentration of $3 \times 10^{5} / \mathrm{ml}$ in potato dextrose (to guarantee $100 \%$ spore germination), on the upper surface of a single marginal ray floret. Twenty ray florets were incubated for $48 \mathrm{~h}$ at nearly $100 \%$ relative humidity. After $48 \mathrm{~h}$, the disease score was assessed as follows: 0 no visible symptoms; 1 infection limited in inoculation droplet size; 2 lesion extended twice to four times the droplet size; 3 large lesion area but still smaller than half of the ray floret; 4 lesion area larger than half of the ray floret; and 5 complete necrosis.

SNP selection and genotyping

EST database establishment and SNP detection have been performed as described in Fu et al. (2016). SNPs were identified as specific SNPs (only polymorphic in one set of crossing parents, i.e. a single population) and common SNPs (polymorphic in both populations). The origin of SNP markers is indicated in the name. For example, marker WGC10601_843_S1F2 means that this marker is developed from contig10601 of the gerbera EST data set (Fu et al. 2016). The number after the first underscore is the SNP position in the contig. At the end of each marker's name, the source of polymorphism is indicated by population (and parent). If it is a specific SNP, it will be followed only with either S or F (S after the second underscore means polymorphic in both $\mathrm{S}$ population parents and S1 means only polymorphic in SP1, etc.). Common SNPs are indicated with both an S and $\mathrm{F}$ in the name. In this case, S1F2 means this marker is a common marker which is polymorphic in parents $\mathrm{S} 1$ and F2 and can be found under an identical name in both maps.

Genotyping of selected reliable SNP markers in parents and all individuals of the two populations was performed by KBioscience (current name LGC genomics) using KASP technology. The genotyping data were visualised in SNPviewer (LGC genomics) to check the segregation type in each population, and SNP markers with segregation type 1:1 and 1:2:1 were included for genetic mapping. SNP makers segregating in a non-Mendelian inheritance pattern were analysed by hypothesising one or more null allele present. After checking the goodness of fit to possible segregation types, these markers were rescored and included.

\section{Genetic linkage map construction}

The genotyping data of two gerbera populations were coded following the population type CP (crosspollinating) in JoinMap® 4.1 (van Ooijen 2006). After created maternal and paternal population nodes, grouping of markers was based upon the test for independence LOD score with a threshold of 4 . Genetic map 
construction used regression mapping and the Kosambi mapping function. Integrated linkage maps of the parental maps were constructed based on the bridge markers $(<h k x h k>$ type marker). Consensus linkage groups of the two populations were constructed with identical common markers segregating in both populations and numbering was named consistent between linkage maps.

\section{QTL analysis}

The means of disease score for each individual on whole inflorescence, bottom and ray floret tests in the two populations were used independently as phenotypic data for QTL analysis. QTL analysis for Botrytis resistance was performed in separate parental linkage maps using MapQTL® 6 (van Ooijen 2009). First, interval mapping was used to find QTL regions associated to each of the traits tested. Based on the result of interval mapping, MQM (multiple QTL models) mapping was performed with the maximum likelihood mixture model using the closest markers as co-factors. Significance LOD thresholds were determined by 1000 permutations corresponding to a genome-wide confidence level of $P<0.05$.

\section{Results}

SNP selection and genotyping results

Gerbera cDNA reads were clustered and assembled into 36,770 EST contigs within which a large number of specific and common SNPs were detected in the parents from the two populations (Fu et al. 2016). For genotyping in the population S, a set of 677 polymorphic SNPs markers, including 477 SNPs common to both populations and 200 specific SNPs, was selected. Similarly, there were 675 SNPs markers selected for population F, including 477 common markers and 198 specific SNPs.

A summary of segregation type for all SNP markers in both populations is shown in Table 1. Of all the selected SNP markers, $68 \%$ were successfully genotyped by KASP in population S and $72 \%$ were successful in population F (Fig. S1a-c illustrate the three visualised segregation results in SNPviewer). A number of markers showing a single group call are considered as non-polymorphic (Fig. S1d); these include 166 SNPs in population $\mathrm{S}$ and 147 in population F. Markers showing scattered segregation without clear grouping were noted as not-fitting segregation (Fig. S1e). The percentages of markers showing a not-fitting pattern were $7 \%$ in population $\mathrm{S}$ and $6 \%$ in population $\mathrm{F}$.

In a number of cases in both the $\mathrm{S}$ and $\mathrm{F}$ populations (Table 1, null allele), parental genotype scores do not seem to fit the found offspring genotypes. These segregating SNP markers could be further analysed assuming the presence of null alleles. For example, in marker WGC19112, the genotype of two parents are A:G (P1) and $\mathrm{G}: \mathrm{G}(\mathrm{P} 2)$, respectively, and the expected segregation in progeny should be $[\mathrm{A}: \mathrm{G}]:[\mathrm{G}: \mathrm{G}]=1: 1$, but the visualised genotyping result in SNPviewer (Fig. S1f) showed three genotype cluster plots [A:A]:[A:G]:[G:G] $\approx 1: 1: 2$ (74:69:133). The possible explanation is the presence of a null allele in P2 (G:Ø) and the actual progeny segregation is $[\mathrm{A}: \mathrm{G}]:[\mathrm{A}: Ø]:[\mathrm{G}: \mathrm{G}]:[\mathrm{G}: \varnothing] \approx 1: 1: 1: 1$, because the genotyping technology cannot distinguish the genotype G:G and G:Ø (they are in the same cluster), also the P2 genotype $\mathrm{G}: \varnothing$ is recognised as G:G.

To use the marker information, we rescored these markers, like WGC19112, with the consideration that both parents are heterozygous. However, information content differed between the parents for such a marker. P1 is heterozygous and WGC19112 is used as a fully informative <lmxll> marker (a). Both A:G and A:Ø offspring clusters are rescored as "lm" and G:G (in fact containing [G:G] and [G:Ø]) as "ll". P2 is also heterozygous and WGC19112 is here regarded as <nnxnp> marker (b). However, only offsprings within the groups A:A and A:G are informative for this parent (group A:A scored as "nn" and group A:G as "np"), the mixed group $\mathrm{G}: \mathrm{G}$ (containing [G:G] and [G:Ø]) is discarded. To distinguish the two ways of scoring, we added a letter "a" or "b" at the end of the marker. Markers in which null alleles were demonstrated with an a and $b$ at the end were mapped at almost the same position on the integrated maps, but in eight markers, sufficient linkage was only found in linkage groups of the most informative parent and not in the other parent.

\section{Linkage map construction}

Both maternal and paternal maps of the two populations were constructed, as well as integrated maps per population and a consensus map of the two populations. There were 30, 29, 27 and 28 linkage groups constructed in SP1, SP2, FP1 and FP2, respectively (Table S1). Total marker number ranged from 259 in parent FP2 to 
Table 1 Overview of the genotyping results of selected SNPs marker

\begin{tabular}{lllllll}
\hline Population & $\begin{array}{l}\text { Markers segregating } \\
\text { in both parents }\end{array}$ & $\begin{array}{l}\text { Markers segregating in } \\
\text { P1 (seed parent) only }\end{array}$ & $\begin{array}{l}\text { Markers segregating in } \\
\text { P2 (pollen parent) only }\end{array}$ & $\begin{array}{l}\text { Markers with } \\
\text { a null allele }\end{array}$ & $\begin{array}{c}\text { No polymorphism } \\
\text { Segregation } \\
\text { not fitting }\end{array}$ \\
\hline S & $135 / 20 \%$ & $159 / 23 \%$ & $126 / 19 \%$ & $41 / 6 \%$ & $166 / 25 \%$ & $50 / 7 \%$ \\
F & $107 / 16 \%$ & $230 / 34 \%$ & $116 / 17 \%$ & $34 / 5 \%$ & $147 / 22 \%$ & $41 / 6 \%$ \\
\hline
\end{tabular}

350 in parent FP1. The observed parental map lengths varied from 1103 to $1498 \mathrm{cM}$ and the average marker distance varied from 3.50 to $4.41 \mathrm{cM}$ per parental map (Table S1).

Parental linkage maps could be aligned via the presence of bridge markers ( $<\mathrm{hkxhk}>$ type markers) that are segregating from both parents (Table S2). Based on the position of the bridge markers, marker order on parental maps showed good consistency, but the distance between the markers on parental linkage maps varied as can be expected. For instance, on maternal linkage group FP1_08, the distance between the markers WGC9125 and WGC18021 is $7.6 \mathrm{cM}$, while the distance on the paternal linkage group (FP2_08) is $12.9 \mathrm{cM}$ (Fig. S2a). By using these bridge markers, the two parental linkage maps could be combined into one integrated linkage map with the same linkage group number code (see Fig. S2a).

Similarly, with the help of common markers, identical parental linkage maps of both crosses could be also be identified and aligned. For instance, there are around 35 markers in linkage group SP1_01, of which some markers are also found in two linkage groups in the parents of the F population (i.e. common markers) indicating that these linkage groups are homologues of SP1_01. So, these fragments are named as FP1_01.1 and FP1_01.2 and as FP2_01.1 and FP2_01.2. The same situation happens on SP1_03, SP2_03, SP1_12, SP2_12, etc. (Table S1). Few maternal and paternal linkage groups (e.g. SP1_21, SP2_23 and FP1_17, FP1_24, FP2_16, FP2_20) could not be aligned to a linkage group of another parent because just a single bridge marker was present or there was a lack of informative common markers (Tables S1 and S2).

These parental linkage maps, with a total of 285 common markers present, can be integrated into a consensus map (see Fig. S2b). In total, 24 consensus linkage groups were merged (Fig. 1, Table S3). As is described in Table S3, the consensus linkage map of 687 SNPs covered $1601 \mathrm{cM}$. The marker density on the consensus map varied from $1.32 \mathrm{cM}$ on linkage group
09 (LG09) to $5.16 \mathrm{cM}$ on LG17, with an average density of $2.57 \mathrm{cM}$. There were 14 gaps larger than $15 \mathrm{cM}$ observed in the consensus linkage map.

Phenotypic traits evaluation for Botrytis resistance

Phenotypic data of resistance to $B$. cinerea were assessed in three tests (whole inflorescence, bottom and ray floret). Histograms of disease testing, resulting from these three traits in the mapping populations $\mathrm{S}$ and $\mathrm{F}$ and indicating transgressive segregations are shown in Fig. 2. The means of the phenotyping data in population $\mathrm{S}$ for whole inflorescence, bottom and ray floret were $2.42 \pm 0.55,2.96 \pm 0.63$ and $2.98 \pm 0.79$. Means in population $\mathrm{F}$ were $3.64 \pm 0.40,3.80 \pm 0.40$ and $3.14 \pm 0.80$, respectively. Based on the skewness and kurtosis scale of the distribution curves, all three tests in the two populations were considered as approximately normally distributed and no transformation of data was performed for QTL analysis.

Disease index of the three disease tests in both populations was analysed by Pearson correlation (Table S4). The coefficients of whole inflorescence and bottom tests in both populations showed moderately high correlations $(R=0.83$ in population $\mathrm{S}, R=0.67$ in population $\mathrm{F})$, but no significant correlation was found to the ray floret tests.

\section{QTL analysis}

QTL analysis was first performed on the four parental linkage maps individually. The genome-wide $(\mathrm{GW})$ LOD significance thresholds $(P<0.05)$ for whole inflorescence, bottom and ray floret were obtained using a permutation test (Table 2). Markers, with LOD scores above the GW threshold in every QTL after interval mapping (IM), were chosen as co-factors for multiple QTL models mapping (MQM mapping). Significant QTLs detected from the four parents are shown in Table 2 and Fig. S3a-c. 


\section{LG01}

-1.2 WGC22294_267_S2a $\left.\begin{array}{c}-1.2 \\ 0.0\end{array}\right] \quad\left[\begin{array}{l}\text { WGC22294_267_S2a } \\ \text { WGC22294_267_S2b }\end{array}\right.$ $4.5] \bigoplus$ WGC35295_167_S

$\left.\begin{array}{l}8.2 \\ 8.7\end{array}\right]$ WGC8621_386_S1

9.3 - WGC36295_149_SF2

9.6 WGC3920_511_S2F1

13.2 1 -WGC28002_228_SF1b

13.8

13.8

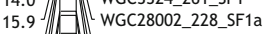

18.2 G WGC30639_405_F1b

$\left.\begin{array}{l}18.7 \\ 19.3\end{array}\right]$ - WGC27835_141_S2F1

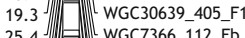

25.4 $]$ 目 W WGC7366_112_Fb $_{\text {WGC33749_426_F2b }}$

26.1 日 WGC7366_426_F2

29.1

35.7

36.0

40.6

40.6
41.6
41.7 WGC 33749_426_F1 WGC33749_426_F2a WGC25286_478_S2F WGC9062_263_S2Fa WGC224_115_SF1b WGC8203_378_S2b WGC8203_378_S2a WGC17612_204_F1a WGC3957_170_SF WGC27987 180 S WGC27470 200 S1F1 WGC9062 263 S2 WGC22__115_SF1 WGC27738_319_S1F1 WGC9062 263 S2Fb WGC8436_166_S2b WGC8870_374_F2 WGC17612 204 F1 WGC9954_900_F2 WGC10214_71_S2F1 WGC18050 562 SF1 WGC5230_1586_S2F2 WGC8140_84_S2 WGC16204_523_S2F1 WGC9128_148_S1F1 WGC20788_411_S1F1 WGC3029_209_S1F1 WGC19258_230_SF1 WGC16204_523_S2F1 WGC16204_523_S2F1 WGC30999 868 S1F1 WGC2920 160 S F1 WGC17781 676 SF1 WGC1781_676_SF1 WGC36485_147_S2F WGC16787_905_S2F1 WGC21895_782_S1F1 WGC3411_200_S1F2 WGC18375_600_S WGC16835_1142_F2 WGC2851_117_S2F2 WGC8870_374_F2b WGC7767_115_Fa WGC5263_310_S1 WGC7767_115_Fb WGC27086 382 S2F1 WGC8436_166_S2a WGC10531_163_S1F2 WGC34062_300_F2 WGC33025_223_F2b WGC33025_223_F2 WGC7745_871_S2 WGC19616_554_S1F2 WGC8430 469 S1F1 WGC 8430-469_STF1 WGC32669_336_SF1 WGC20122_505_S2 WGC10226_238_Fb WGC729_629_S2 WGC2655_394_S2 WGC10226_238_Fa WGC36541_224_SF1 WGC19529_158_SF1
LG02

-1.7 [WGC11464_359_S1F $\left.\begin{array}{r}-1.7 \\ 0.0 \\ 21.9\end{array}\right] \|\left[\begin{array}{l}\text { WGC11464_359_S1F } \\ \text { WGC6199_1191_S2F2 } \\ \text { WGC8508_520_S2 } \\ \text { WGC18942_274_S1F1b } \\ \text { WGC18942_274S1F1 }\end{array}\right.$ $22.3]$ WGC18942_274_S1F1 23.8 WGC18942 274 S1F1 25.3] WGC34976_88_S

28.6] - -WGC19660_424_S2F2b 29.3] WGC19660_424_S2F2a

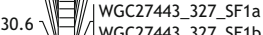
1.6. WGC27443_327_SF1b 30.9 - WGC4477_1171_SF1 31.0 OWGC34745_323_S2F 31.2 _ WGC3004_110_SF1 1.3

41.2 46.0

69.6

70.4

\section{LG05}

0.07.

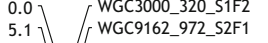
5.5 WGC25114_277 SF

6.1 - WGC4138 154 SF

6.5 . WGC31215 552

6.9 DGC11108_178_S

7.0 [WGC4253_92_S1

\section{LG03}

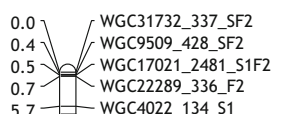

5.7 - WGC4022_134_S1

15.8 WGC3283_124_F

18.6 WGC16203_309_S1F

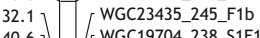

$47.3]\left[/\left[\begin{array}{l}W G C 19704 \_238 \_S 1 \\ W G C 18569 \_197 \_S 1\end{array}\right.\right.$

48.0] $]$ - $\left[\begin{array}{l}\text { WGC18569_197_S1 } \\ \text { WGC27578_1176 S1 }\end{array}\right.$

48.5 $]$ - $\left[\begin{array}{l}\text { WGC27578_1176_S1 } \\ \text { WGC27091_180_F1 }\end{array}\right.$

48.5 $]$ - WGC27091_180_F1

49.7 $]$ W WGC11409_325_SF2

51.4 WGC22443 342 S1F2

55.1 WG W6073 123 S2a

56.2 WGC6821 571 S1F2

56.2 $=$ WGC6821_571_S1

56.7 WGC1546_255_S

56.8 E

56.9 $\int$ 目 $\begin{aligned} & \text { WGC12914_1020_S } \\ & \text { WGC8380_543_S1F2 }\end{aligned}$

56.9 WGC8380_543_S1

$57.1=-\left(\begin{array}{l}W G C 7722 \_846 \_S 1 \\ \text { WGC11924_433_S1 } \\ \text { WGC12078_2206_S1 }\end{array}\right.$

WGC19080_240_S1F1

57.2 W WGC20720 497_S1F2

57.7

WGC5979_360_S1F2

WGC14842 414 Sb

WGC14842_414_S

WGC14842_414_Sa

WGC4082_303_S2

WGC2825_374_S2

WGC8867_154_S1F2

WGC2630_188_S2

WGC17399_462_S2

WGC3917_184_S1F2

WGC27213_976_F2

WGC849_141_F

WGC 8825292 S1F

WGC6073_123 S2b

WGC3317_107_F1

WGC20799_220_S1F1

WGC8442_332_S1

WGC5809_222_F1
WGC26837_145_S

\section{LG04}

0.0 WGC19498_261_S1F1

0.9 WGC3574_475_SF

$\left.\begin{array}{l}11.4 \\ 39.0\end{array}\right] \cap\left[\begin{array}{l}\text { WGC20870_99_S1F1 } \\ \text { WGC21560_189_S1F }\end{array}\right.$

40.2 WGC21560_189_S1Fa

45.4 WGC18155_189 S

46.8 WGC3228_98_S1F1

47.5 WGC2952_358_S1F

50.4 WGC19682_206_S2F1

55.0 WGC16724_245_F1a

56.7 WGC16724_245_F1

$\left.\begin{array}{l}56.7 \\ 57.2\end{array}\right]$. W WGC31490_172_S2F2

6.3 $]$ WGC18033_534_S1F1

62.4 WGC18837_429_S2a

62.5

6.7 |WGC8153_138_F1

62.7 ) ( WGC27713_180_S2F2

$62.8 \sqrt{\text { WGC2732_157_F1 }}$

63.1 WGC6488_560_S2

63.2 WGC8372_105_S2F1

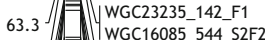

63.9 的 $=\left[\begin{array}{l}\text { WGC16085_544_S2F2 } \\ \text { WGC19120_131_S2F1 }\end{array}\right.$

69.6 WGC9403_546_S1F1

70.0 WGC9403_546_S1F1a

70.6 WGC25129_300 S2b

70.8 WGC25129 300 S2a

71.6 - WGC35912_221_SF1

76.1 WGC18737_810_S

76.5 U WGC34449_558_S

77.6 - WGC3259_328_S

89.4 -WGC9403_546_S1F1b

95.3 丹 WGC25081_216_S

108.2 لUWGC30016_79_SF2

114.7 $\left[\begin{array}{l}\text { WGC2621_159_S2 } \\ \text { WGC30016_79_SF2 }\end{array}\right.$

118.0 WWC15966_1511_S2F2

\section{LG07}

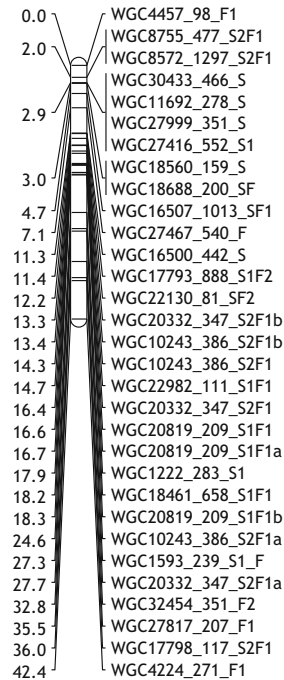

\section{LG06}

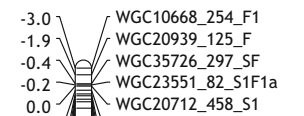

Fig. 1 Consensus linkage map of gerbera 


\section{LG08}

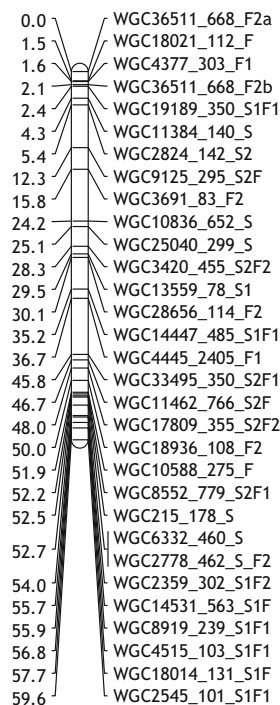

\section{LG12}

$\left.\begin{array}{l}0.0 \\ 0.1\end{array}\right] \quad\left[\begin{array}{l}\text { WGC18656_631_S2F1b } \\ \text { WGC8201_354_S2F1b }\end{array}\right.$ 1.4 WGC14466_1718_F2 4.5 肺 WGC8201_354_S2F1a

4.7 1 - WGC8201_354_S2F1a

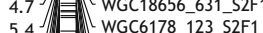

5.6 $]=$ WGC18030_324_S2F

5.8

5.9 EGC18656_631_S2F1 WGC27945_490_S2F1 WGC17525_192_SF

WGC18765_397_S1F1 WGC27726_205_S2F1 WGC26751 388 S2F WGC3269_135_SF2

WGC26730_306_S2F1

|WGC26751_388_S2F1b WGC26751_388_S2F1 WGC19515_468_SF1 WGC19515 468 SF1 WGC19515 468_SF1a WGC35820_247_F WGC9243_208_S1F1 WGC3872_221_S1b WGC9121_215_S1F2 WGC771_772_S1F2

WGC34091_249_S1F2 WGC3872_221_S1a WGC19432_94_S1F1 WGC4376_224_S1F2 WGC34695_116_F2 WGC34695_116_F2 WGC4217_100_S2F1 WGC8837_493_S2F1 WGC24795 328 SF1 -WGC3758_76_S WGC33479_427_S1F WGC25045_123_SF2 -WGC27464_56_SF -WGC27464_56_SF WGC9301_544_S1F2b WGC9301_544_S1F2a -WGC9301_544_S1F2 WGC10305_190_F WGC20619_638_S1F1 WGC16523_281_F1b WGC4149_407_S1F2 WGC693_624_F1 WGC4460_246_F1 WGC16523_281_F1a

\section{LG09}

0.0 WGC27639_103_F1 0.2 WWGC35370_146_S2F1 0.7 WGC22285_206_S2F1b 0.9 WGC18994_594_S2F 1.5
1.7 2.4 目 $W$ WGC17528_144_F1 2.7 $]$ 目 5.0 WGC27903_379_S2F1b 7.6

\section{LG13}

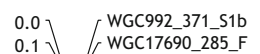

WGCC17690_285_F

= WGC18819_128_S2F1a

4.0

4.6 目 $\left[\begin{array}{l}\text { WGC5574_460_S2F1 } \\ \text { WGC27846_452_S2F1 }\end{array}\right.$

4.8

6.7

8.1

8.4
9.3

9.3
11.0
12.0

12.0
12.5

14.3

$\left.\begin{array}{l}14.5 \\ 19.9\end{array}\right]$ WGC3108_124_SF1

32.4 WGC11627_252_S2F2

46.0 [ WGC18668_127_F1b

51.6 WWC18668 127 F1a

56.8 [WGC19078_418_S1F1

\section{LG16}

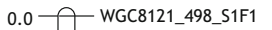

\section{LG10}

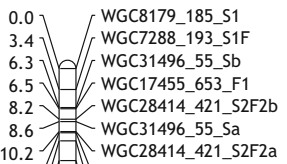

10.2 - WGC28414_421_S2F2a $\left.\begin{array}{l}10.4 \\ 11.7\end{array}\right]\left[\begin{array}{l}\text { WGC4521_2140_S1F1b } \\ \text { WGC10920 210_F2 }\end{array}\right.$ 14.9 -WGC4521_2140_S1F1a 18.7 $\Rightarrow$ WGC4521_2140_S1F1 23.2 D WGC23649_136_S2F1 28.2 W WGC8772_188_S

28.4 $]$ 二 (WGC19637_457_S2F1 28.7 WGC26459_452_S 29.0 WGC25934_157_S1F 29.1 31.8 目 WGC7561 567F 37.4 37.4 . WGC3850 37.6 WGC3850_171_F2a 49.8 50.5 50 52.7 53. 55. 55.7 57.7 60.9 64.2 69. 71.8

\section{LG14}

WGC34723 249 F2b 0.0 $\left.\begin{array}{ll}\text { WGC34723_249_F2b } & 0.0 \\ \text { WGC34723_249_F2 } & 3.6\end{array}\right] \quad\left[\begin{array}{l}\text { WGC36216_955_F1 } \\ \text { WGC1697_224_51 }\end{array}\right.$

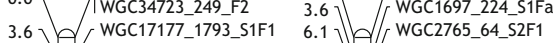
4.8 -WGC3507_143_F 9.2 WGC1697_224_S1F 6.4- WGC4358_306_S2 9.4 -WGC1697_224_S1Fb 8.5-WGC3801 506_S W WGC10483 460_S2F1 10.6 7 WGC22208 450S 9.5 J

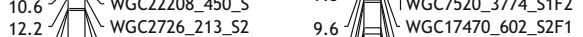

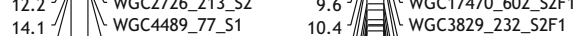
$\left.\begin{array}{l}14.1 \\ 14.4\end{array}\right]\left(\begin{array}{ll}\text { WGC4489_77_S1 } & 10.4 \\ \text { WGC7302 742_S1F2 } & 12.7\end{array}\right] \Rightarrow \equiv\left(\left[\begin{array}{l}\text { WGC3829_232_S2F1 } \\ \text { WGC4126_149FF1 }\end{array}\right.\right.$ $\left.\left.\begin{array}{l}14.4 \\ 27.2\end{array}-\begin{array}{ll}\text { WGC7302_742_S1F2 } & 12.7 \\ \text { WGC4355_157_S2F1 } & 13.1\end{array}\right]\right)=\left(\begin{array}{l}\text { WGC4126_149_F1 } \\ \text { WGC36235_369_S1F1 }\end{array}\right.$ 45.4 [ [ WGC18204_532_F2 48.6] WGC6974_151_S2F2 52.0] WGC17277_213_S1F1

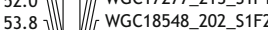
$55.0]$ ] - $-(\mid$ WGC36346_215_S 56.9] - WGC11531_363_S2F1 57.1 WE WG9176_170_SF1 58.4 - WGC17320_229_S2F2 58.6 - WGC2015_590_S2 59.1 WGC10429_697_S2F2 59.2 WGC19617_114_F $\left.\begin{array}{l}59.7 \\ 60.4\end{array}\right]$ WGC17277_213_S1F1

LG17
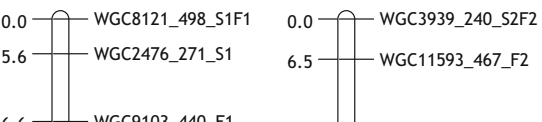

16.6 WGC9103_440_F1

-WGC18703_968_S1F1 $\left.\begin{array}{l}36.8 \\ 54.5\end{array}\right]\left[\begin{array}{l}\text { WGC7576_107_S1F1 } \\ {[\text { WGC15379_306_S2 }}\end{array}\right.$

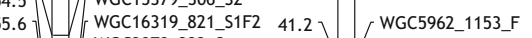
6.9 WGC3979_333_S WGC8562_422_S WGC7599_395_S2 WGC889 106 S2 WGC8760_289_S2F1 $61.1 \sqrt{ }$ 目 WGC17081_316_S2F1

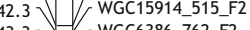
43.3 = WGC6386_762_F2 44.5 队 WGC8417_313_F2 45.3
46.5 WGC3585_238_F2

\section{LG11}

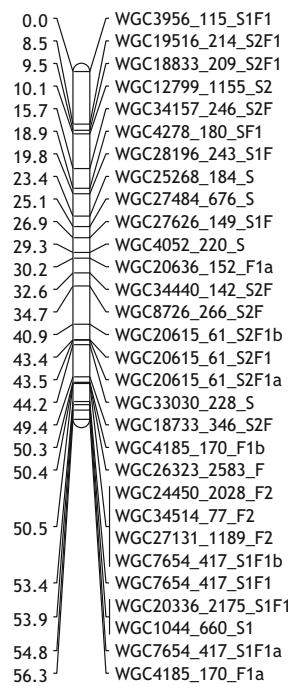

13.1

18.2

19.8

Fig. 1 (continued) 

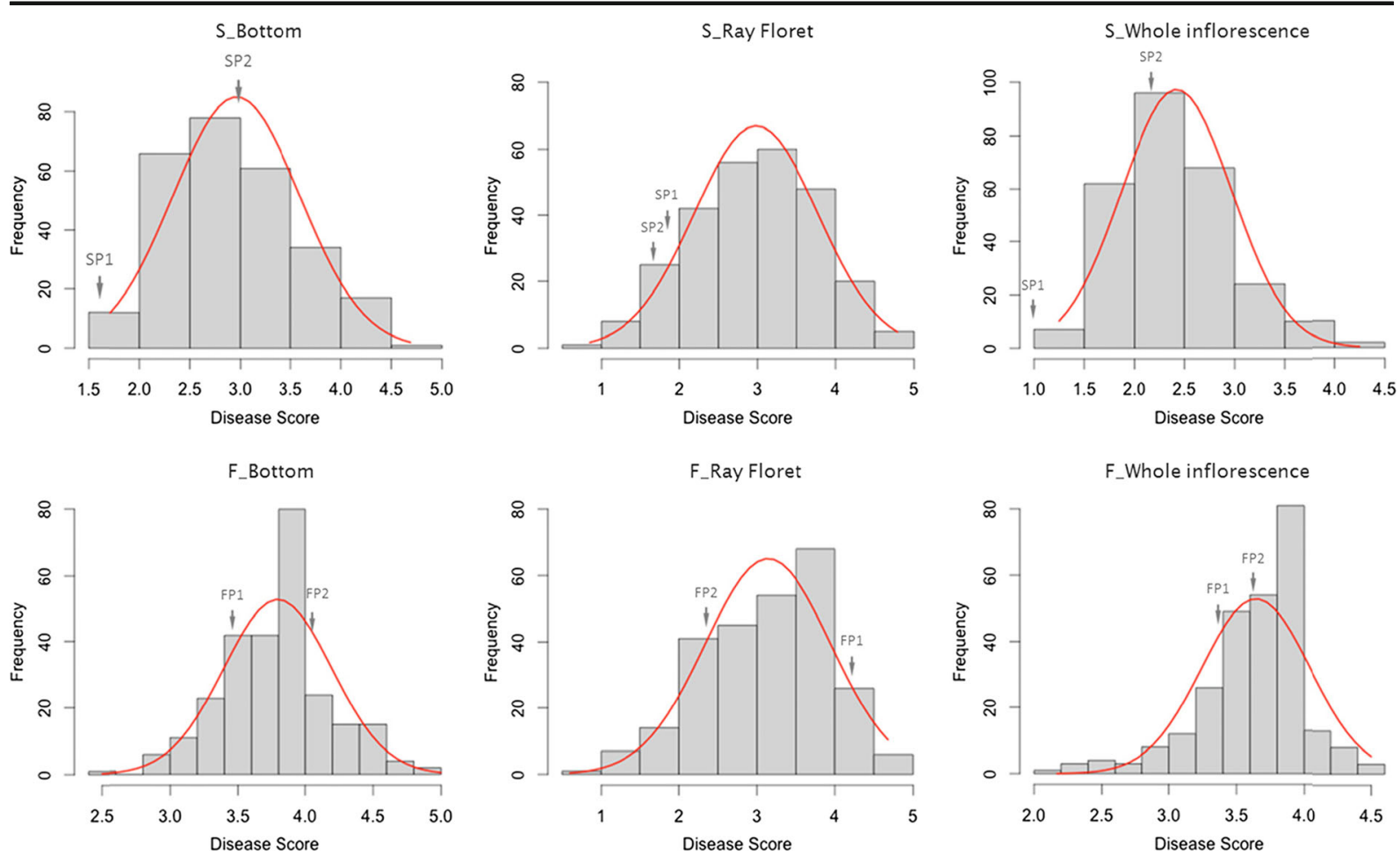

Fig. 2 Disease index distribution of $S$ and $F$ populations in bottom, ray floret and whole inflorescence, respectively. Normal distribution curves are shown above the histograms in red. Arrows indicate the disease score of parents

In the S population, seven significant QTLs for Botrytis resistance were detected by MQM mapping and 13 QTLs in the F population. The difference in numbers of QTLs found between the two populations is defined by the number of QTLs associated with Botrytis resistance in ray floret. There is only one ray floret QTL found in the S population but seven in the F population (Table 2). Phenotypic variance explained by single QTLs ranged between 5.7 and $11.4 \%$, with three QTLs (RBQB4, RBQWI4 and RBQWI6) higher than $10 \%$. Three QTLs, RBQWI2, RBQWI4 and RBQWI6 from SP1, FP1 and FP2, respectively, were found on LG23 at similar positions in the consensus map (see Fig. S4) indicating this may be a single QTL. Interestingly, a QTL for whole inflorescence and bottom (RBQB3 and RBQWI3) shared an identical position with marker WGC18733_346_S2F on LG11 of population $\mathrm{S}$.

Several QTLs were detected on both parental linkage groups separately and showed overlapping positions on the integrated linkage group, like RBQWI1 from SP1 and RBQWI3 from SP2 on LG11 and RBQB5 from FP1 and RBQB6 from FP2 on LG9. In these cases, alleles from both parents contributed to the resistance in the progeny. We identified the favourable and unfavourable alleles from the parents of these QTLs. Progeny can then be divided into four groups: progeny with the presence of two favourable alleles $(+/+)$, with one favourable allele from one of the parents $(+/-$ or $-/+)$ and no favourable allele present $(-/-)$. The mean disease score of each progeny group for each QTL is shown in Table 3. The mean disease scores of individuals with two favourable alleles $(+/+)$ were all significantly lower than those for individuals with no favourable allele present (-/-) and also show advantage over individuals with one favourable allele only.

\section{Discussion}

Genetic linkage mapping and integration

In this study, we constructed the first gerbera genetic linkage maps from two F1 populations using newly generated SNP markers. Genetic linkage map construction for cultivated ornamental crops often use F1 populations (Debener and Mattiesch 1999; Han et al. 2002; Rajapakse et al. 2001; Shahin et al. 2011; Zhang et al. 
Table 2 QTLs found for whole flower, bottom and petal test in the parental genotypes of both populations

\begin{tabular}{|c|c|c|c|c|c|}
\hline \multirow[t]{2}{*}{ QTL } & \multirow[t]{2}{*}{ Parents } & \multirow[t]{2}{*}{ Flanking markers } & \multirow[t]{2}{*}{ LG } & \multicolumn{2}{|l|}{ MQM } \\
\hline & & & & $\begin{array}{l}\text { LOD } \\
(\mathrm{GW})\end{array}$ & $\% \operatorname{expl}$ \\
\hline RBQB1 & SP1 & WGC11243_647_S2F1a & 2 & $4.4(4.0)$ & 6.3 \\
\hline RBQB2 & SP1 & WGC2476_271_S1 & 16 & $4.6(4.0)$ & 6.6 \\
\hline RBQB3 & $\mathrm{SP} 2$ & WGC18733_346_S2F & 11 & $4.6(4.1)$ & 7.6 \\
\hline RBQB4 & FP1 & WGC16204_523_S2F1 & 1 & $6.8(4.0)$ & 10.3 \\
\hline RBQB5 & FP1 & WGC28102_213_S2F1 & 9 & $4.5(4.0)$ & 7.5 \\
\hline RBQB6 & FP2 & WGC18158_119_F1b & 9 & $4.8(3.9)$ & 8 \\
\hline RBQRF1 & SP2 & WGC17798_117_S2F1 & 7 & $5.3(4.0)$ & 8.9 \\
\hline RBQRF2 & FP1 & WGC22343_292_SFa & 5 & $6.5(4.1)$ & 8.6 \\
\hline RBQRF3 & FP1 & WGC35370_146_S2F1 & 9 & $4.8(4.1)$ & 6.2 \\
\hline RBQRF4 & FP1 & WGC828_408_S2F & 15 & $6.1(4.1)$ & 8 \\
\hline RBQRF5 & FP1 & WGC35264_283_S2F1 & 18 & $5.9(4.1)$ & 7.6 \\
\hline RBQRF6 & FP2 & WGC7520_3774_S1F2 & 15 & $4.9(4.0)$ & 7 \\
\hline RBQRF7 & FP2 & WGC6074_441_S2F & 18 & $4.0(4.0)$ & 5.7 \\
\hline RBQRF8 & FP2 & WGC9226_226_F2 & 21 & $4.75(4.0)$ & 8.0 \\
\hline RBQWI1 & SP1 & $\begin{array}{l}\text { WGC1044_660_S1, } \\
\text { WGC33030_228_S }\end{array}$ & 11 & $4.8(4.0)$ & 7.3 \\
\hline RBQWI2 & SP1 & WGC407_4995_S1F1 & 23 & $5.3(4.0)$ & 8.2 \\
\hline RBQWI3 & SP2 & WGC18733_346_S2F & 11 & $5.2(4.1)$ & 8.6 \\
\hline RBQWI4 & FP1 & WGC1084_721_F & 23 & $6.8(4.1)$ & 11.1 \\
\hline RBQWI5 & FP2 & WGC5962_1153_F & 17 & $5.6(4.0)$ & 8.3 \\
\hline RBQWI6 & FP2 & WGC22447_285_Fb & 23 & $7.6(4.0)$ & 11.4 \\
\hline
\end{tabular}

Name of QTLs are RBQ (as Resistance Botrytis QTL) followed by the initials of disease tests used. Null alleles are marked with a letter 'a' or ' $b$ ' in the end

$B$ Bottom, $P F$ ray floret, $W I$ whole inflorescence test, $L G$ indicates linkage group and the LG number in the two populations, $G W$ indicates genome-wide significant threshold level $P<0.05 ; \%$ expl. is the percentage of total variance explained by the QTL

Table 3 Difference between the mean score of individuals with the presence of two, one or no favourable allele from the parents

\begin{tabular}{|c|c|c|c|c|c|}
\hline \multirow{3}{*}{$\begin{array}{l}\text { QTLs } \\
\text { Flanking } \\
\text { markers }\end{array}$} & \multirow{3}{*}{$\begin{array}{l}\text { Bottom } \\
\text { RBQB5 + RBQB6 } \\
\text { WGC28102_213_S2F1 }\end{array}$} & \multicolumn{2}{|l|}{ Ray floret } & \multicolumn{2}{|l|}{ Whole inflorescence } \\
\hline & & RBQRF4 + RBQRF6 & RBQRF5 + RBQRF7 & RBQWI1 + RBQWI3 & RBQWI4 + RBQWI6 \\
\hline & & WGC828_408_S2F & WGC35264_283_S2F1 & WGC33030_228_S & WGC1084_721_F \\
\hline & WGC18158_119_F1b & WGC7520_3774_S1F2 & WGC6074_441_S2F & WGC18733_346_S2F & WGC22447_285_Fb \\
\hline Genotype $^{1}$ & Mean \pm S.E. & Mean \pm S.E. & Mean \pm S.E. & Mean \pm S.E. & Mean \pm S.E. \\
\hline$+/+$ & $3.621 \pm 0.044^{\mathrm{a} 2}$ & $2.840 \pm 0.099^{\mathrm{a}}$ & $2.732 \pm 0.126^{\mathrm{a}}$ & $2.244 \pm 0.060^{\mathrm{a}}$ & $3.436 \pm 0.057^{\mathrm{a}}$ \\
\hline$+/-$ & $3.847 \pm 0.041^{\mathrm{bc}}$ & $3.328 \pm 0.090^{\mathrm{b}}$ & $3.152 \pm 0.071^{\mathrm{b}}$ & $2.440 \pm 0.067^{\mathrm{b}}$ & $3.631 \pm 0.043^{\mathrm{b}}$ \\
\hline$-/+$ & $3.764 \pm 0.058^{\mathrm{b}}$ & $3.299 \pm 0.096^{\mathrm{b}}$ & $3.152 \pm 0.071^{\mathrm{b}}$ & $2.316 \pm 0.057^{\mathrm{ab}}$ & $3.739 \pm 0.050^{\mathrm{bc}}$ \\
\hline$-1-$ & $3.938 \pm 0.052^{\mathrm{c}}$ & $3.414 \pm 0.121^{\mathrm{b}}$ & $3.430 \pm 0.115^{\mathrm{b}}$ & $2.635 \pm 0.074^{\mathrm{c}}$ & $3.776 \pm 0.041^{\mathrm{c}}$ \\
\hline
\end{tabular}

\footnotetext{
${ }^{1}+/+$ represents individuals with the presence of two favourable alleles from both parents; $+/-$ represents individuals carrying one favourable from P1 and one unfavourable allele from P2; -/+ represents individuals carrying one unfavourable allele from P1 and favourable from P2; $-/-$ represents individuals with the presence of the two unfavourable alleles from both parents

${ }^{2}$ mean of each groups with letter $\mathrm{a}, \mathrm{b}$ and $\mathrm{c}$ shows significant difference $(P<0.05)$
} 
2010) because many ornamental plants, including gerbera, are outcrossing species with complex genetic backgrounds and high heterozygosity that cannot be easily selfed due to serious inbreeding depression effects.

Four parental genetic linkage maps were constructed by using SNP markers from EST data (Fu et al. 2016). Most selected SNP markers showed a Mendelian segregation in the populations. For some loci, the allele segregation and allele ratios indicated the presence of null alleles. Three flanking markers of a QTL contained null alleles. These markers with null alleles probably come from mutations in the marker region which in the RNAseq data analysis of Fu et al. (2016) may have led to assembly of these sequences in alternative contigs and thus stayed unnoticed during SNP identification. We scored markers with null alleles for each parent separately in order to use the marker data as much as possible and found these two-way scored markers are mapped on almost the same positions in the integrated map and the consensus map. The fact that the four alleles of these markers are all different reflects the complex genetic background of gerbera.

Based on the location of the bridge (<hkxhk> type) markers on the maternal and paternal linkage maps, we found the markers' order on parental maps shows a good consistency, but the distance between the markers on parental linkage maps varies. This is caused by independent meiotic events occurring in the two heterozygous parents and the different frequency of recombination determines the location of markers in each parent (Gebhardt 2007). This also explains the difference in linkage group length between the parents of a cross. Markers common to both populations could be used to merge maps between the two populations and to arrive at a consensus map which was helpful for comparisons between the two populations in QTL mapping. From the integrated and consensus maps, we notice that some parental chromosomes appeared as separate (fragmented) linkage groups in one genotype whereas they were in one LG in another genotype (e.g. FP1_01.1 and FP1_01.2 vs SP1_01). Fragmentation also occurs in integrated maps of single populations. Generally, this occurs more often in the F population ( 9 out of 20 integrated LGs) than in the S population (6 out of 21 integrated LGs). This might be due to a lower number of markers in FP2. Given the offspring numbers in both populations, a theoretical minimum marker distance of $0.4 \mathrm{cM}$ is possible. So, by introducing more markers, map quality may be further improved.
For gerbera, we expected 25 linkage groups $(2 n=50)$. However, a total of 24 consensus linkage groups could be established. There are no additional linkage groups left in any of the four parental maps which could be assigned to LG25. This could be related to the size of this particular chromosome and the number of markers used in our study. Introducing higher numbers of markers might result in retrieving LG25. Also, a lack of polymorphism between alleles of this chromosome could cause the inability to find this linkage group.

\section{Gerbera grey mould phenotyping}

Gerbera grey mould occurs mainly on gerbera capitulum in the production and post-harvest process. Different symptoms in infected gerbera cultivars were found, either necrotic spots on ray and trans florets or rot on disc florets. The mechanism underlying plant resistance against $B$. cinerea is not well understood, but it is generally accepted that plant resistance to this necrotrophic pathogen is quantitative and polygenic (Poland et al. 2009; Rowe and Kliebenstein 2008; St. Clair 2010). In a structured mapping population, quantified disease indexes after inoculation can be used to analyse plant responses to this pathogen and perform QTL mapping. However, there is no standard bioassay approach for evaluating plant resistance to $B$. cinerea available.

Previous studies on Arabidopsis and tomato (AbuQamar et al. 2006; Denby et al. 2004; Ferrari et al. 2007; Finkers et al. 2008; Finkers et al. 2007b; Hu et al. 2013; Rowe and Kliebenstein 2008; ten Have et al. 2007; Zhang and Van Kan 2013) are mainly based on infection assays using drop inoculation or spray inoculation with conidia suspension on leaves or stems, then measuring the lesion expansion rate, lesion size or camalexin accumulation. In gerbera, leaf and stem infections are of little importance and mainly flower infections lead to losses. To thoroughly assess the disease severity on gerbera flowers, we developed spray-inoculation tests on whole inflorescences (whole inflorescence and bottom), as well as a droplet-inoculation test for single ray florets. The tests were devised as simple tests in which a large number of flowers and ray florets could be tested in a relatively short period of time to avoid season influences during the testing period.

As a necrotrophic pathogen, Botrytis relies primarily on its abilities to kill the host plant cell and subsequently decompose the plant tissue and consume it for its own growth (van Kan 2006). The fungus can use different 
infection paths in the complex organs that capitulum are and from experiments with cultivar panels, different responses between cultivars were observed and these led to the three different tests used. Among the three tests, there is a high correlation in both populations between the infection data of whole inflorescence and bottom disease symptoms. Apparently, the mechanism of defence within the chosen parents of the two crosses is more similar with regard to these two traits compared to the wider set of cultivars used in the development of the tests. The test on ray florets (petal) is clearly different from the other two tests. Therefore, for the ray floret test versus whole inflorescence and bottom, it can be envisaged that different genes are involved in resistance to Botrytis. Similarly, ten Have et al. (2007) also observed that resistance to Botrytis on tomato leaves and stems is distinct from each other.

QTLs mapping and analysis

QTLs detected varied between the two populations and also between the three tests. The reason for the lower number of QTLs found in the S population for ray floret resistance might be the small difference in ray floret disease score between the two parents of this population. The two $\mathrm{F}$ population parents, by contrast, showed a large difference in disease score for ray floret. Three QTL regions for whole inflorescence test, which were detected on different parental maps separately, co-localised on linkage group 23 for both populations. Although the QTL region still spans $20 \mathrm{cM}$ on the consensus map between the most significant loci, the flanking bridge markers indicated possible overlapping of parental linkage groups and the existence of favourable alleles.

A relative high correlation between bottom and whole inflorescence was found in both populations, yet there is only one identical locus in both populations showing a significant QTL in both tests. More common QTLs for whole inflorescence and bottom might be expected given the correlation between the two tests. Apparently, not all QTLs underlying the high correlation of the two tests can be detected which could be due to a lack of resolving power to detect minor QTLs for both disease indexes at the same time in a population. Environmental variance between test weeks may influence both tests in a similar way; however, numbers of repetitions per week were too low to be able to study this.

In this study, several QTLs with minor effect for Botrytis resistance on gerbera inflorescences were detected. The results showed that, similar to Botrytis resistance in other plants, defence against $B$. cinerea on gerbera is quantitative and genetically complex, with probably the involvement of different infection mechanisms (Denby et al. 2004; Finkers et al. 2007a; Rowe and Kliebenstein 2008). QTLs found in our study may seem minor-effect QTLs, which are more difficult to use in breeding programs than major-effect QTLs or single resistance genes. However, several QTLs detected from separate parental linkage groups were found in overlapping locations on the integrated map and we assume that these correlative QTLs are probably from a common gene with positive and negative alleles which can be defined as quantitative trait alleles (QTAs, Schäfer-Pregl et al. 1998). With the presence of two positive QTAs, gerbera resistance to Botrytis increased significantly.

For two reasons, we think there is potential for introgression of favourable QTL alleles in breeding to increase resistance to Botrytis in gerbera: (a) phenotyping Botrytis disease is difficult and the large environmental component in testing has a downsizing effect on the total explained variance found in QTLs, i.e. contributions of QTLs to genetic variance explained will be higher and (b) compared to the disease tests (conditions chosen to avoid effects of incidence), the disease pressure in commercial greenhouses will be much lower and environmental conditions are less favourable for Botrytis infection (Finkers et al. 2007b). Under such conditions, the effect of the QTLs may be much stronger.

This mapping study provides the first genetic map of gerbera and by using SNP markers derived from EST sequences (Fu et al. 2016), a generally useable framework is provided which can be used for other studies as well and provides the first step in unravelling the complexity of the genetic background of resistance to Botrytis in gerbera.

Acknowledgements Open access funding provided by Wageningen University. We are thankful for the support from the Foundation Technological Top Institute Green Genetics (3CFL030RP) and Jan van Kan for providing us with the Botrytis strain and useful advice in the start-up phase of disease testing.

Open Access This article is distributed under the terms of the Creative Commons Attribution 4.0 International License (http:// creativecommons.org/licenses/by/4.0/), which permits unrestricted use, distribution, and reproduction in any medium, provided you give appropriate credit to the original author(s) and the source, provide a link to the Creative Commons license, and indicate if changes were made. 


\section{References}

AbuQamar S et al (2006) Expression profiling and mutant analysis reveals complex regulatory networks involved in Arabidopsis response to Botrytis infection. Plant J 48:2844. doi:10.1111/j.1365-313X.2006.02849.x

Bastiaan-Net S, Balk P, Roelofsen C, van Wordragen M, Mes J (2007) Developing marker genes for the prediction of Botrytis cinerea infection on flower petal of Rosa hybrida. Paper presented at the 14th International Botrytis Symposium, Cape Town, South Africa

Clark CA, Lorbeer JW (1975) The role of phenols in Botrytis brown stain of onion. Phytopathology 65:338-341

Debener T, Mattiesch L (1999) Construction of a genetic linkage map for roses using RAPD and AFLP markers. Theor Appl Genet 99:891-899. doi:10.1007/s001220051310

Denby KJ, Kumar P, Kliebenstein DJ (2004) Identification of Botrytis cinerea susceptibility loci in Arabidopsis thaliana. Plant J 38:473-486. doi:10.1111/j.1365-313x.2004.02059.x

Elad Y, Pertot I, Cotes Prado AM, Stewart A (2016) Plant hosts of Botrytis spp. In: Fillinger S, Elad Y (eds) Botrytis - the fungus, the pathogen and its management in agricultural systems. Springer International Publishing, Cham, pp 413486. doi:10.1007/978-3-319-23371-0 20

Ferrari S, Galletti R, Denoux C, De Lorenzo G, Ausubel FM, Dewdney J (2007) Resistance to Botrytis cinerea induced in Arabidopsis by elicitors is independent of salicylic acid, ethylene, or jasmonate signaling but requires PHYTOALEXIN DEFICIENT3. Plant Physiol 144:367-379. doi:10.1104 /pp.107.095596

Finkers R, Finkers R, van Heusden AW, Meijer-Dekens F, van Kan JAL, Maris P, Lindhout P (2007a) The construction of a Solanum habrochaites LYC4 introgression line population and the identification of QTLs for resistance to Botrytis cinerea. Theor Appl Genet 114:1071-1080. doi:10.1007/s00122-0060500-2

Finkers R, van den Berg P, van Berloo R, ten Have A, van Heusden AW, van Kan JAL, Lindhout P (2007b) Three QTLs for Botrytis cinerea resistance in tomato. Theor Appl Genet 114:585-593. doi:10.1007/s00122-006-0458-0

Finkers R et al (2008) Quantitative resistance to Botrytis cinerea from Solanum neorickii. Euphytica 159:83-92. doi:10.1007/s10681007-9460-0

Fu Y, Esselink GD, Visser RGF, Van Tuyl JM, Arens P (2016) Transcriptome analysis of gerbera hybrid: including in silico confirmation of defence genes found. Front Plant Sci 7. doi:10.3389/fpls.2016.00247

Gebhardt C (2007) Molecular markers, maps and population genetics. In: Potato biology and biotechnology. Elsevier Science B.V, Amsterdam, pp 77-89. doi:10.1016/B978044451018-1/50047-6

Glazebrook J (2005) Contrasting mechanisms of defense against biotrophic and necrotrophic pathogens. Annu Rev Phytopathol 43:205-227. doi:10.1146/annurev.phyto.43.040204.135923

Han T-H, van Eck H, de Jeu M, Jacobsen E (2002) The construction of a linkage map of Alstroemeria aurea by AFLP markers. Euphytica 128:153-164. doi:10.1023/a:1020921103374

Hansen HV (1999) A story of the cultivated gerbera. New Plantsman 6:85-95
Hu P, Zhou W, Cheng ZW, Fan M, Wang L, Xie DX (2013) JAV1 controls jasmonate-regulated plant defense. Mol Cell 50: 504-515. doi:10.1016/j.molcel.2013.04.027

Kerssies A (1993a) Horizontal and vertical-distribution of airborne conidia of Botrytis cinerea in a gerbera crop grown under glass. Neth J Plant Pathol 99:303-311. doi:10.1007 /Bf01974311

Kerssies A (1993b) Influence of environmental conditions on dispersal of Botrytis cinerea conidia and on post-harvest infection of gerbera flowers grown under glass. Plant Pathol 42:754-762. doi:10.1111/j.1365-3059.1993.tb01562.x

Kliebenstein DJ, Rowe HC, Denby KJ (2005) Secondary metabolites influence Arabidopsis/Botrytis interactions: variation in host production and pathogen sensitivity. Plant J 44:25-36. doi:10.1111/j.1365-313X.2005.02508.x

Leroux P (2007) Chemical control of Botrytis and its resistance to chemical fungicides. In: Elad Y, Williamson B, Tudzynski P, Delen N, Leroux P (eds) Botrytis: biology. Pathology and Control, Springer, Dordrecht, pp 195-222. doi:10.1007/9781-4020-2626-3_12

Mengiste T, Chen X, Salmeron J, Dietrich R (2003) The BOTRYTIS SUSCEPTIBLE1 gene encodes an R2R3MYB transcription factor protein that is required for biotic and abiotic stress responses in Arabidopsis. The Plant Cell Online 15:2551-2565

Poland JA, Balint-Kurti PJ, Wisser RJ, Pratt RC, Nelson RJ (2009) Shades of gray: the world of quantitative disease resistance. Trends Plant Sci 14:21-29. doi:10.1016/j. tplants.2008.10.006

Prins TW, Wagemakers L, van Kan JAL (2000) Structure and expression in planta of Botrytis cinerea ubiquitin genes. Eur J Plant Pathol 106:693-698. doi:10.1023/A:1008713230963

Rajapakse S, Byrne DH, Zhang L, Anderson N, Arumuganathan K, Ballard RE (2001) Two genetic linkage maps of tetraploid roses. Theor Appl Genet 103:575-583. doi:10.1007/P100002912

Rowe HC, Kliebenstein DJ (2008) Complex genetics control natural variation in Arabidopsis thaliana resistance to Botrytis cinerea. Genetics 180:2237-2250. doi:10.1534/genetics.108.091439

Salinas J, Verhoeff K (1995) Microscopical studies of the infection of gerbara flowers by Botrytis cinerea. Eur J Plant Pathol 101:377-386. doi:10.1007/bf01874851

Schäfer-Pregl R et al (1998) Analysis of quantitative trait loci (QTLs) and quantitative trait alleles (QTAs) for potato tuber yield and starch content. Theor Appl Genet 97:834-846. doi:10.1007/s001220050963

Shahin A et al (2011) Genetic mapping in Lilium: mapping of major genes and quantitative trait loci for several ornamental traits and disease resistances. Plant Breed 130:372-382. doi:10.1111/j.1439-0523.2010.01812.x

St. Clair DA (2010) Quantitative disease resistance and quantitative resistance loci in breeding. Annu Rev Phytopathol 48: 247-268. doi:10.1146/annurev-phyto-080508-081904

ten Have A, Berloo R, Lindhout P, van Kan JL (2007) Partial stem and leaf resistance against the fungal pathogen Botrytis cinerea in wild relatives of tomato. 117:153-166 doi:10.1007/s10658-006-9081-9

Thomma BPHJ, Eggermont K, Penninckx IAMA, Mauch-Mani B, Vogelsang R, Cammue BPA, Broekaert WF (1998) Separate jasmonate-dependent and salicylate-dependent defense-response pathways in Arabidopsis are essential for resistance 
to distinct microbial pathogens. P Natl Acad Sci USA 95: 15107-15111. doi:10.1073/pnas.95.25.15107

Thomma BPHJ, Eggermont K, Tierens KFMJ, Broekaert WF (1999) Requirement of functional ethylene-insensitive 2 gene for efficient resistance of Arabidopsis to infection by Botrytis cinerea. Plant Physiol 121:1093-1101. doi:10.1104 /pp.121.4.1093

van Baarlen P, Legendre L, van Kan JAL (2007) Plant defence compounds against botrytis infection. In: Elad Y, Williamson B, Tudzynski P, Delen N (eds) Botrytis: biology, pathology and control. Springer Netherlands, Berlin Heidelberg New York, pp 143-161. doi:10.1007/978-1-4020-2626-3_9

van Kan JAL (2006) Licensed to kill: the lifestyle of a necrotrophic plant pathogen. Trends Plant Sci 11:247-253. doi:10.1016/j. tplants.2006.03.005

van Ooijen JW (2006) JoinMap ${ }^{\circledR}$ 4, Software for the calculation of genetic linkage maps in experimental populations

van Ooijen JW (2009) MapQTL ® 6, Software for the mapping of quantitative trait loci in experimental populations of diploid species
Williamson B, Tudzynsk B, Tudzynski P, van Kan JAL (2007) Botrytis cinerea: the cause of grey mould disease. Mol Plant Pathol 8:561-580. doi:10.1111/J.13643703.2007.00417.x

Zhang LS, Van Kan JAL (2013) Botrytis cinerea mutants deficient in D-galacturonic acid catabolism have a perturbed virulence on Nicotiana benthamiana and Arabidopsis, but not on tomato. Mol Plant Pathol 14:19-29. doi:10.1111/j.13643703.2012.00825.x

Zhang F, Chen SM, Chen FD, Fang WM, Li FT (2010) A preliminary genetic linkage map of chrysanthemum (Chrysanthemum morifolium) cultivars using RAPD, ISSR and AFLP markers. Sci Hortic-Amsterdam 125:422-428. doi:10.1016/j.scienta.2010.03.028

Zhang W, Kwon ST, Chen F, Kliebenstein DJ (2016) Isolate dependency of Brassica rapa resistance QTLs to Botrytis cinerea. Front Plant Sci 7. doi:10.3389/Fpls.2016.00161 